\title{
Criminologie
}

\section{La couverture du crime par la presse : un portait fidèle ou déformé ?}

\section{Thomas Gabor et Gabriel Weimann}

Volume 20, numéro 1, 1987

Faire les nouvelles : journalisme et affaires criminelles

URI : https://id.erudit.org/iderudit/017247ar

DOI : https://doi.org/10.7202/017247ar

Aller au sommaire du numéro

\section{Éditeur(s)}

Les Presses de l'Université de Montréal

ISSN

0316-0041 (imprimé)

1492-1367 (numérique)

Découvrir la revue

Citer cet article

Gabor, T. \& Weimann, G. (1987). La couverture du crime par la presse : un portait fidèle ou déformé ? Criminologie, 20(1), 79-98.

https://doi.org/10.7202/017247ar
Résumé de l'article

The authors examined, through content analysis, some criticisms that have been levelled at the press in its coverage of crime. The propositions examined included the accusations that newspapers are preoccupied with violence and "street" crime, that they focus on the bizarre, are superficial in their reporting of crime, misinform the public about the characteristics of offenders and victims, and exhibit a conservative bias in their analysis.

This study of a major Canadian daily newspaper revealed substantiation for some of these claims but failed to support others. Violent and street crimes received disproportionate coverage and very few articles contained an in-depth analysis of the roots of crime or the workings of the criminal justice system. The evidence was less clear or non-existent in relation to the claims that the press focus on nonroutine events, that they provide distorted images of offenders and victims and that they have a conservative bent.

Commentant l'impact des masse-médias, Marshall McLuhan (1978) soulignait:

To invade the private person, or to invade a group with teaching, with doctrines, with entertainment, all these are alike forms of violence. To assume the right to program the sensibilities or thoughts and fantasies of individuals or groups, has long been taken for granted as a viable form of personal or social action...

Today, however, there is a new dimension in all of these activities. Electric media move information and people at the speed of light. It is this instant and total quality that constitutes the condition of mass man and the mass society ( $p$. 212).
Tous droits réservés @ Les Presses de l'Université de Montréal, 1987
Ce document est protégé par la loi sur le droit d'auteur. L’utilisation des services d’Érudit (y compris la reproduction) est assujettie à sa politique d'utilisation que vous pouvez consulter en ligne.

https://apropos.erudit.org/fr/usagers/politique-dutilisation/ 


\section{LA COUVERTURE DU CRIME PAR LA PRESSE: UN PORTRAIT FIDĖLE OU DÉFORMÉ?** \\ Thomas Gabor** Gabriel Weimann***}

The authors examined, through content analysis, some criticisms that have been levelled at the press in its coverage of crime. The propositions examined included the accusations that newspapers are preoccupied with violence and "street" crime, that they focus on the bizarre, are superficial in their reporting of crime, misinform the public about the characteristics of offenders and victims, and exhibit a conservative bias in their analysis.

This study of a major Canadian daily newspaper revealed substantiation for some of these claims but failed to support others. Violent and street crimes received disproportionate coverage and very few articles contained an in-depth analysis of the roots of crime or the workings of the criminal justice system. The evidence was less clear or non-existent in relation to the claims that the press focus on nonroutine events, that they provide distorted images of offenders and victims and that they have a conservative bent.

Commentant l'impact des masse-médias, Marshall McLuhan (1978) soulignait:

To invade the private person, or to invade a group with teaching, with doctrines, with entertainment, all these are alike forms of violence. To assume the right to program the sensibilities or thoughts and fantasies of individuals or groups, has long been taken for granted as a viable form of personal or social action...

Today, however, there is a new dimension in all of these activities. Electric media move information and people at the speed of light. It is this instant and total quality that constitutes the condition of mass man and the mass society (p.212).

Ce qu'il appelle «la violence des médias» a au moins trois ramifications fondamentales dans un contexte criminologique. D'abord on peut avancer que les médias structurent et encouragent certaines attitudes du

\footnotetext{
* Ce texte a été traduit par Pierre Pinsonneault.

** Thomas Gabor est professeur au département de criminologie à l'Université d'Ottawa.

***Gabriel Weimann est professeur à l'University de Haifa en Isarel.
} 
public envers la criminalité et le système de justice qui vont dans le sens des conceptions officielles du crime et des remèdes à y apporter (Halloran, 1978). En second lieu on peut également considérer qu'un public mal informé par les médias aura tendance à favoriser des politiques criminelles pour le moins inappropriées. Des études ont en effet démontré que les évaluations et les opinions du public reflètent beaucoup plus les conceptions que se font les médias du problème de la criminalité que la réalité elle-même (Davis, 1952; Graber, 1980). Enfin les médias peuvent avoir un rôle à jouer dans les conflits et les comportements agressifs entre les individus ou entre les groupes. Bien que dans ce dernier domaine les renseignements soient contradictoires, il semble cependant se dégager qu'il y ait une relation entre la violence des médias et les comportements agressifs (Andison, 1977; Eysenck et Nias, 1978; Garofalo, 1980).

On a déjà isolé un certain nombre de facteurs qui sous-tendraient des conceptions erronées et une certaine pathologie sociale que l'on attribue souvent aux médias. On a ainsi avancé qu'autant dans les médias électroniques que dans les imprimés on accordait une couverture journalistique beaucoup trop large aux phénomènes de violence et ce autant dans les nouvelles que dans les variétés (Garofalo, 1981). En plus du fait qu'il y a abondance de violence dans les médias, on s'accorde généralement sur le fait qu'ils mettent un accent disproportionné sur les crimes de violence négligeant ceux qui sont commis contre la propriété ou l'ordre public, tout cornme ceux qui le sont par des hommes publics, des sociétés et de façon générale par des gens d'un statut social élevé (Reid, 1971; Surgeon General's Scientific Advisory Committee, 1971; Howitt et Cumberbatch, 1975; Jones, 1978; Royal Commission, 1976; Dominick, 1978, Halloran, 1978; Sherizen, 1978; Dussuyer, 1979; Graber, 1980). On remarque d'autre part que le nombre de crimes de différentes catégories que l'on retrouve dans les médias n'a quelquefois qu'une faible ressemblance avec ce qui se produit dans la réalité (Jones, 1976). Davis (1952), Antunes et Hurley (1977), ainsi que d'autres ont signalé la très faible association, quelquefois même inversée, entre le nombre de crimes rapportés sur la scène locale par un journal donné et le nombre réel de crimes commis dans cette région. Fishman (1978) a même été jusqu'à faire allusion à des «vagues de crime artificielles" créées par les médias pour satisfaire aux besoins d'un journal ou d'un réseau de télévision.

Une autre catégorie de distorsions, que l'on a remarquée, implique les caractéristiques réciproques des victimes et des criminels. Bien que dans ce domaine nous ne possédons pour le moment que peu de données, quelquefois même contradictoires, il ressort cependant que les médias décrivent souvent victimes et criminels comme beaucoup plus âgés qu'ils 
ne le sont en réalité (Pandiani, 1978; Graber, 1980). Dans les émissions de variétés à la télévision, on a remarqué par ailleurs que la classe sociale et la race auxquelles appartiennent les délinquants, different considérablement de ce que l'on retrouve dans les statistiques officielles (Pandiani, 1978). En représentant aussi faussement les caractéristiques des victimes et des criminels, on peut contribuer à la formation de stéréotypes ou plus encore renforcer certains stéréotypes déjà existants (Surgeon General's Committee, 1971). On peut également développer des stéréotypes de situation, c'est le cas de programmes de télévision où la défense de la loi implique essentiellement l'usage de la force (Dominick, 1978).

On a également accusé les différents médias de faire du sensationnalisme en mettant l'accent sur le spectaculaire [i.e. les crimes de haine (heinous crime)], sur ce qui était inhabituel, qui sortait de la routine. Dussuyer (1979), après une étude d'une quarantaine de journaux ontariens, constate que le peu d'articles qui s'intéressent aux femmes criminelles sont cependant plus longs et mis plus en évidence que ceux qui couvrent des criminels mâles. Dans un sens, cependant, c'est ce genre d'événement qui peut être considéré comme une «nouvelle» (Roshco, 1975). En dépit d'une telle définition, l'emphase qui est mise sur des choses qui sortent de l'ordinaire ne peut que rendre inévitable une fausse représentation de la réalité dans le domaine du reportage judiciaire.

On souligne également souvent que ce genre de reportage est souvent très superficiel (Isaacs, 1961; Roshco, 1975); Halloran, 1978) et qu'en mettant l'accent sur les côtés sensationnels et stimulants des nouvelles, les médias ne s'arrêteront qu'occasionnellement sur les causes profondes du comportement criminel et du contexte social où il fermente. Les médias ignorent également souvent les développements reliés à une affaire après l'arrestation comme par exemple la décision de poursuivre, le plea bargaining et l'impact qu'a le système correctionnel sur l'individu (Dominick, 1978). Les journalistes qui s'intéressent au crime ont d'autre part la fâcheuse tendance de manquer d'esprit critique face aux informations qu'ils reçoivent de leurs sources (tout d'abord la police) et des statistiques officielles en général.

Puisqu'elles sont surtout basées sur des sources officielles d'information, les nouvelles concernant les crimes ont un biais conservateur, qui tend à refléter les définitions et les explications établies sur le problème tout comme les pratiques établies pour y répondre. À cause de leur tendance à exagérer l'ampleur des phénomènes de violence et les désordres sociaux, les médias contribuent également à conditionner favorablement le public à l'usage de la force (Halloran, 1978). 
Cette attitude conservatrice se retrouve également présente quand les médias tentent de voir à qui incombe la responsabilité dans un crime. Ainsi, dans une étude qui a porté sur les comptes rendus de trois journaux torontois sur une vague d'attaques violentes contre des femmes en 1982, Vomvakis et Ericson (1984) ont remarqué que dans presque la moitié des cas, les journalistes ou leurs sources considéraient la victime responsable. L'ensemble des autres articles référait au rôle de l'infracteur ou au mauvais fonctionnement du système de justice criminelle. Un peu moins de $10 \%$ des textes mettaient l'accent sur des problèmes structurels dans la société...e.g., l'aliénation entre hommes et femmes. Graber (1980), dans son étude sur le Chicago Tribune, a également fait état du fait qu'on ne retrouvait pas dans ce journal des appels en faveur de changements profonds dans la société en vue de combattre la criminalité.

On peut avancer un certain nombre de faits pour expliquer les lacunes dans les reportages de situations criminelles tout comme on peut tenter de comprendre pourquoi les programmes de divertissement nous donnent une représentation déformée du crime et des criminels.

Il faut d'abord considérer le fait qu'en Amérique du Nord les médias appartiennent à des particuliers qui les gèrent. En ce sens, ils sont très sensibles au marché et tentent d'abord d'accroitre leur circulation ou leur cote d'écoute (Epstein, 1973). Dans ce but on mettra l'accent sur les événements les plus sensationnels, ceux qui auront comme effet de maximiser le nombre de lecteurs ou de téléspectateurs. On peut également atteindre le même objectif en couvrant les aspects les plus passionnants d'une histoire criminelle - habituellement les circonstances de l'acte lui-même, plutôt que faire une analyse édifiante des facteurs qui sous-tendent l'événement ou encore d'expliquer la réaction du système de justice criminelle à un tel acte. L'importance accordée au compte rendu, plutôt qu'à l'analyse dans un journal ou un poste de télévision est fonction de politiques différentielles qui de leur côté sont dessinées en fonction d'un marché particulier.

La relation symbiotique qui existe entre beaucoup de reporters qui se spécialisent dans le crime et la police explique également pourquoi les médias ont tendance à accorder tant d'importance à la couverture de crimes violents, de crimes de rue et pourquoi aussi ils ont tendance à adopter des points de vue sur les problèmes criminels qui sont ouvertement simplistes et favorables au statu quo (Sherizen, 1978). En s'alignant étroitement sur la police, le journaliste est ainsi assuré d'avoir accès à une information facile à comprendre et régulière sur les tendances générales du crime, tout comme sur les détails particuliers à certains incidents. Du même coup, la police bénéficie d'une antenne pour promouvoir ses vues 
sur le crime et pour en même temps faire valoir ses besoins en ressources supplémentaires (Molotch et Lester, 1978). Il ne faut cependant pas perdre de vue que quand l'information émane de ces seules sources officielles elle se limite aux seuls crimes connus et colligés par la police.

Cette vulnérabilité des médias face à une masse d'information provenant de la police est aggravée par le fait que les reporters spécialisés dans les histoires criminelles semblent très sensibles à l'influence policière et peu enclins à adopter une attitude critique dans leurs reportages en général. En effet, ces journalistes sont souvent moins scolarisés que leurs confrères, ils sont souvent plus âgés, occupent leur fonction pendant plus longtemps, ont des vues plus conservatrices et peuvent même être moins bien payés et avoir un statut inférieur à celui de leurs pairs (Sherizen, 1978).

Une autre raison qui peut expliquer que les informations sur les problèmes criminets puissent subir une distorsion par rapport à la situation réelle, réside dans la façon de les présenter autant dans les journaux qu'à la télévision ou à la radio. Dans les journaux, par exemple, on n'accorde pas une section spéciale aux nouvelles criminelles comme on le fait pour les informations sportives. Une telle section, bien qu'elle n'assure pas nécessairement une couverture compréhensive, permet quand même au lecteur d'avoir une idée sur l'ensemble des nouvelles chaque jour et permet de couvrir plus en profondeur et de faire des commentaires sur quelques événements ou problèmes majeurs. L'actualité criminelle a tendance à combler les trous et son importance variera en fonction de celle des autres nouvelles d'un jour à l'autre. Comme enfin elle est dispersée à travers un journal, on ne retrouve pas de synthèse cohérente de la situation à un moment donné et peu fréquemment une étude de problèmes importants.

\section{L'ÉTUDE}

Dans notre étude nous avons voulu examiner la validité de certaines des critiques mentionnées précédemment envers les médias. Pour ce faire nous avons procédé à une analyse de contenu d'articles référant à la criminalité dans le plus important quotidien d'Ottawa, The Citizen. Les articles et éditoriaux que nous avons retenus ont été publiés dans The Citizen entre le premier décembre 1984 et le 31 janvier 1985. En sélectionnant des articles et des éditoriaux nous voulions également déterminer les proportions respectives d'analyses et de comptes rendus factuels. C'est à partir de mots clés tels "crime", "criminels", "arrestation", «inculpation» et de catégories de délits que l'on retrouve dans le code criminel canadien que nous avons isolé des articles de journaux relatifs au crime. 
Un de nos premiers objectifs était de déterminer la proportion d'articles consacrée aux crimes commis dans la région par rapport à ceux effectués en dehors de la région d'Ottawa. Le résultat de cette mesure nous permettrait de voir si l'image de la criminalité que l'on présente au lecteur ressemble à celle à laquelle il peut être confronté à Ottawa. Ces images furent par la suite analysées de plusieurs façons.

Nous avons d'abord examiné quels types de crime étaient couverts par les articles afin de vérifier l'hypothèse qui avance que les médias sont d'abord préoccupés par des crimes violents et accordent peu d'attention à la criminalité "non traditionnelle» comme celle commise par les compagnies. Nous avons ensuite effectué des comparaisons avec la distribution actuelle des différents types de délits à Ottawa, cela afin de voir quels crimes étaient sous ou surreprésentés dans les colonnes de The Citizen. À cette fin, nous avons utilisé les données colligées par la police d'Ottawa.

Nous avons ensuite regardé l'âge et le sexe des suspects et des victimes pour voir jusqu'à quel point l'image qui se dégageait des médias correspondait à la réalité. Pour ce faire nous avons utilisé les données de la police et les rapports de victimisation. Nous avons également tenu compte du sexe du reporter pour voir si, comme l'affirment certaines recherches antérieures, ils sont en grande majorité de sexe masculin.

Nous avons ensuite utilisé deux instruments de mesure pour nous permettre de voir où ces articles donnent l'impression de situer la responsabilité primaire dans l'incident (Weimann, Gabor et Brault, 1985). Le premier instrument mesure la couverture journalistique faite sur la victime par rapport à celle faite sur l'offenseur en nombre de lignes dans un article donné. On divise celles consacrées à l'offenseur par le nombre de celles où on parle de la victime. Une seconde technique était plus explicite dans le sens qu'elle consistait à déterminer à qui, de l'offenseur ou de la victime, on attribuait la responsabilité de l'incident, et cela à partir des écrits des reporters et des dires de leurs sources. À cause du fait que le rôle joué par l'offenseur dans l'événement serait sûrement mis en évidence dans la plupart des comptes rendus et que mesurer les rôles respectifs de l'offenseur et de la victime pourrait être une opération fort subjective, nous avons alors décidé de réaliser cette opération de mesure en mettant l'accent sur la victime. Plus spécifiquement, nous avons recherché des références à la contribution de la victime. Ainsi tout article dans lequel le comportement rapporté de la victime était relié à l'incident soit dans le temps soit encore à cause de la qualité de "victime» était considéré comme un article ou on mettait en évidence la contribution de la victime à l'incident. Quoique cette façon de voir les choses peut n'impliquer qu'une faible contribution de la victime, le message commu- 
niqué aux lecteurs pouvait être très différent. Une alternative aurait été de classer la participation de la victime sur une échelle de responsabilité; ce qui est loin d'être une tâche facile. Comme notre objectif ne visait qu'à déterminer le pourcentage d'articles à partir desquels le lecteur pouvait raisonnablement conclure que la victime avait joué un rôle quelconque dans sa propre victimisation, nous ne nous sommes pas arrêté à cette dernière possibilité qui impliquait des notions juridico-philosophiques concernant la responsabilité.

Nous avons ensuite pris note de l'âge et du sexe autant du délinquant que de la victime, tout comme nous avons également considéré le sexe du reporter, pour voir si ces variables affectaient d'une part le taux de couverture entre victime et délinquant et d'autre part pour voir également si elles jouaient un rôle dans la possibilité que certains articles fassent référence à la contribution de la victime à l'incident.

Nous avons enfin étudié les articles que nous considérons analytiques afin de déterminer si, comme certaines recherches l'ont déjà constaté, ils s'inspiraient d'une vision conservatrice des causes de la criminalité et suggéraient des politiques en ce sens.

L'analyse de contenu fut effectuée par deux codeurs indépendants. Les différences dans le codage furent solutionnés par des consultations entre eux et, quand cela s'avéra nécessaire, avec les chercheurs.

\section{LES RÉSULTATS}

Compte tenu de nos critères de choix nous avons retenu 500 articles. De ce nombre $93 \%$ étaient d'ordre factuel et le reste consistait soit en éditoriaux soit en articles de nature analytique. Presque les trois quarts de l'ensemble de nos articles rapportaient des événements d'ordre criminels qui s'étaient déroulés à l'extérieur de la région d'Ottawa. On peut donc en déduire que l'image générale que le lecteur du The Citizen se fait du crime, c'est celle d'incidents qui se sont passés ailleurs qu'à l'endroit où il réside.

Dans le tableau 1, nous pouvons comparer la distribution des crimes dont il a été fait mention dans "The Citizen" avec la distribution réelle des crimes à Ottawa. Autant pour les articles dans leur ensemble que pour ceux qui ne s'intéressent qu'aux nouvelles locales, on remarque une nette surreprésentation des crimes de violence, particulièrement l'homicide. Plus du tiers de l'ensemble des articles et presque le cinquième de ceux qui s'intéressent aux nouvelles locales ont trait à l'homicide, alors que, selon les données de la police d'Ottawa, moins de une demi de $1 \%$ des infractions au Code criminel ont trait au meutre ou aux tentatives de 
TABLEAU 1

Délits rapportés dans «The Citizen" et distribution réelle de la criminalité à Ottawa*

\begin{tabular}{lccc}
\hline Délits & $\begin{array}{c}\text { \% d'articles } \\
\text { tous les crimes }\end{array}$ & $\begin{array}{c}\% \text { d'articles } \\
\text { crimes locaux }\end{array}$ & $\begin{array}{c}\text { \% de délits } \\
\text { criminels commis } \\
\text { à Ottawa }\end{array}$ \\
\hline Homicide & 36,4 & 17,9 & 0,04 \\
Assaut & 4,8 & 9,4 & 4,3 \\
Assaut sexuel & 5,2 & 7,7 & 0,6 \\
Vol qualifié & 7,8 & 20,5 & 2,0 \\
Vol par effraction & 1,6 & 5,1 & 20,7 \\
Vol simple & 3,6 & 5,1 & 45,0 \\
Fraude & 1,8 & 0,9 & 5,0 \\
Drogues & 7,2 & 9,4 & 1,0 \\
Organisationnel & 1,0 & - & - \\
Autres & 30,6 & 24,0 & 21,4 \\
& 100,0 & 100,0 & 100,0 \\
\hline
\end{tabular}

* Les données proviennent du rapport annuel du Département de police d'Ottawa pour l'année 1984.

meurtre. D'un autre côté des infractions telles le cambriolage, le vol ou la fraude, sont nettement sous-représentées dans les comptes rendus journalistiques. En effet, alors que ces types d'infractions représentent plus de $70 \%$ de toutes les infractions au Code criminel à Ottawa, elles ne font l'objet que de $7 \%$ des articles pendant la période que nous avons étudiée. Les infractions relatives à la drogue font l'objet, de leur côté, d'une publicité disproportionnée à leur importance réelle.

Les différences les plus dignes de mention entre les nouvelles locales ou extérieures ont trait aux crimes d'homicide, d'assaut, de vol qualifié et de vol par effraction. Les nouvelles de l'extérieur concernaient beaucoup plus souvent des cas d'homicides alors que celles qui s'intéressent à la scène locale couvraient plutôt les assauts, les vols qualifiés et les vols simples.

Le sexe des infracteurs et des victimes dont on fait mention dans les articles, ainsi que celui du reporter apparaissent dans le tableau 2. Le fait que les infracteurs et les victimes dont il est fait état dans les articles soient majoritairement mâles correspond à la réalité. En effet, plus de $90 \%$ des personnes arrêtées à Ottawa pour des délits graves sont des hommes (Gabor et Gottheil, 1984). Cependant, au niveau de la victimisation, la différence entre les sexes semble dans l'ensemble moins accentuée (Conklin, 1981; Solliciteur général du Canada, 1983), ce que l'on retrouve bien dans les articles que nous avons retenus où la propor- 
TABLEAU 2

Sexe des infracteurs, victimes et reporters selon les articles retenus

\begin{tabular}{lccccc}
\hline & $\%$ & $\%$ & $\%$ & $\%$ & $\%$ \\
& Masculin & Féminin & Mixte & lnconnu ou N.A.* & $\begin{array}{c}\% \\
\text { Total }\end{array}$ \\
\hline Infracteur & 65,8 & 3,2 & 6,0 & 25,0 & 100,0 \\
Victime & 28,2 & 16,8 & 8,6 & 46,4 & 100,0 \\
Reporter & 16,2 & 3,4 & - & 80,4 & 100,0 \\
\hline
\end{tabular}

*N.A. - Ne s'applique pas, car l'article était un êditorial.

tion hommes-femmes diminue considérablement d'infracteurs à victimes. Par contre il semble, selon les rapports récents, que le nombre de crimes commis en groupes est de beaucoup supérieur à ce que laisse croire les données de notre étude (la catégorie mixte dans le tableau 2) (Gabor et Gottheil, 1984; Zimring, 1981). Enfin, bien qu'il nous ait été difficile d'obtenir des renseignements précis sur le sexe des reporters, notre étude révèle qu'ils sont encore surtout des hommes (Sherizen, 1978).

Bien que le profil des relations entre l'âge d'une part $\mathrm{e}^{+}$la criminalité ou la victimisation d'autre part varie selon les différentes catégories d'infractions, on remarque cependant une tendance générale, dans les deux cas, à atteindre un sommet vers l'adolescence, tendance qui va s'atténuant avec le vieillissement (Mannheim, 1965; Conklin, 1981; Solliciteur général du Canada, 1983). Le tableau 3 nous indique que les articles de journaux que nous avons examiné rendaient assez bien compte de la réalité dans le domaine des groupes d'âge.

\section{TABLEAU 3}

$\hat{A}$ ge des infracteurs et des victimes selon les articles retenus

\begin{tabular}{|c|c|c|c|c|c|c|c|}
\hline & $\begin{array}{c}\% \\
\text { Moins de } \\
20\end{array}$ & $21-30$ & $\begin{array}{c}\% \\
31-40\end{array}$ & $\begin{array}{c}\% \\
41-50\end{array}$ & $\begin{array}{c}\% \\
51 \text { et } \\
\text { plus }\end{array}$ & $\begin{array}{c}\% \\
\text { Inconnu } \\
\text { ou N.A.* }\end{array}$ & $\begin{array}{c}\% \\
\text { Total }\end{array}$ \\
\hline $\begin{array}{l}\text { Infracteur } \\
\text { Victime }\end{array}$ & $\begin{array}{r}7,0 \\
12.6\end{array}$ & $\begin{array}{r}18,8 \\
5,0\end{array}$ & $\begin{array}{r}11,0 \\
5,0\end{array}$ & $\begin{array}{l}8,0 \\
2,6\end{array}$ & $\begin{array}{l}4,0 \\
6,8\end{array}$ & $\begin{array}{l}51.2 \\
68,0\end{array}$ & $\begin{array}{l}100,0 \\
100,0\end{array}$ \\
\hline
\end{tabular}

*Ne s'applique pas car l'article était un éditorial. 
En effet, on remarque dans ce tableau qu'il y a moins d'infracteurs dans le groupe «3i-40" qu'il n'y en a dans celui des « $21-30$ » et cette baisse continue pour les autres groupes d'âge. Le fait que dans le groupe des moins de 20 ans on remarque une sous-représentation des infracteurs est probablement dû aux contraintes qui obligent les médias à ne pas rendre publics l'identité et les caractéristiques des jeunes délinquants. En ce qui concerne les victimes, le plus grand nombre d'entre elles se situent aux deux extrêmes: moins de vingt ans ou plus de 50. Ici encore on assiste à une diminution de leur importance numérique à travers le vieillissement, le groupe des plus de cinquante ans faisant exception. On peut se demander si l'attention qu'on apporte au problème social que représente la victimisation dans ce groupe d'âge, n'explique pas l'intérêt accru des journalistes pour ces situations.

Nous nous sommes ensuite penchés sur l'ampleur de la couverture journalistique que l'on accordait réciproquement à l'infracteur et à la victime. Comme d'autres recherches l'ont démontré (Graber, 1980), nous avons constaté que l'on s'intéressait beaucoup plus au rôle joué par l'infracteur que par la victime et que, quand les deux attiraient l'attention, le texte consacré à l'infracteur était plus important. Dans le tableau 4 nous voyons que la victime n'est même pas mentionnée dans un cas sur trois et reçoit moins de dix lignes de texte dans un autre tiers des cas. Par contre, l'infracteur de son côté est, sauf dans $4,2 \%$ des cas, toujours mentionné. Il retient même une attention de plus de trente lignes dans presque le tiers des cas.

TABLEAU 4

Étendue de la couverture accordée aux infracteurs et aux victimes

\begin{tabular}{|c|c|c|c|c|c|c|c|c|}
\hline & $\begin{array}{c}\% \\
\text { Pas } \\
\text { mentionné }\end{array}$ & $\begin{array}{c}\% \\
\text { Sous } \\
10 \text { lignes }\end{array}$ & $\begin{array}{c}\% \\
11.20 \\
\text { lignes }\end{array}$ & $\begin{array}{c}\% \\
21-30 \\
\text { lignes }\end{array}$ & $\begin{array}{c}\% \\
31-40 \\
\text { lignes }\end{array}$ & $\begin{array}{c}\% \\
41 \text { et }+ \\
\text { lignes }\end{array}$ & $\stackrel{\%}{\text { N.A. }}{ }^{*}$ & $\begin{array}{c}\text { क } \\
\text { Total }\end{array}$ \\
\hline Infracteur & 4,2 & 24,4 & 21,6 & 13,4 & 14,4 & 15,8 & 6,4 & 100,0 \\
\hline Victime & 33,6 & 33,2 & 11,0 & 6.8 & 5,6 & 3,4 & 6,4 & 100,0 \\
\hline
\end{tabular}

*Ne s'applique pas car l'article étail un éditorial. 
C'est dans une perspective différente que, dans le tableau 5 , nous avons regardé la couverture relative accordée aux infracteurs et aux victimes. Nous nous sommes demandé quel pourcentage d'articles accordait plus d'attention à l'une ou l'autre parties et également quel pourcentage en accordait autant à l'une qu'à l'autre. On peut y observer que dans presque les deux tiers des cas, les infracteurs ont bénéficié de plus d'attention que les victimes qui elles, n'en retiennent plus que dans un peu plus que du sixième des cas. Enfin, c'est dans encore moins de cas qu'elles se sont vues accorder une attention égale à celle des infracteurs par les journalistes.

\section{TABLEAU 5}

Couverure relative accordée aux infracteurs et aux victimes

\begin{tabular}{lc}
\hline & $\%$ d'articles \\
\hline Plus grande couverure à l'infracteur & 62,6 \\
Plus grande couverture à la victime & 17.4 \\
Couverture égale & 13,6 \\
Ne s'applique pas & 6,4 \\
Total & 100.0 \\
\hline
\end{tabular}

*Articles considérés comme analytiques.

Qu'est-ce qui influence le plus l'importance de la couverture journalistique accordée avec infracteurs et aux victimes? On trouvera, dans le tableau 6, des éléments de réponse à cette question puisqu'on y a mesuré la relation de ces deux variables avec le type de crime dont il est fait mention, le sexe de l'infracteur, de la victime et du reporter ainsi que l'âge de l'infracteur et de la victime.

La seule relation statistiquement significative (selon le test du $\mathrm{X}^{2}$ ) est exercée par le sexe de la victime. Cela est dû non pas à des différences entre hommes et femmes (qui sont négligeables) mais plutôt au fait que quand plusieurs personnes des deux sexes sont victimisées l infracteur est plus suceptible de retenir l'attention du journaliste que quand il n'y a qu'une seule victime qu'elle soit homme ou femme.

On a remarqué également un certain nombre de relations, qui bien que non significatives, $n$ 'en sont pas moins intéressantes. Ainsi, les victimes sont plus susceptibles d'obtenir une plus grande couverture journalistique que les infracteurs si le crime est violent par opposition à 
TABLEAU 6

Relation entre la catégorie de crime. le sete, l'âge et l'importance

de la couverture journalistique selon que l'on est infracteur ou victime

\begin{tabular}{|c|c|c|c|c|c|c|c|}
\hline & & $\begin{array}{l}\text { \% de cas où plus } \\
\text { grande couverture } \\
\text { à l'infracteur }\end{array}$ & $\begin{array}{l}\% \text { de cas où plus } \\
\text { grande couverture } \\
\text { à la victime }\end{array}$ & $\begin{array}{l}\% \text { de cas } \\
\text { couverture } \\
\text { égale }\end{array}$ & $\mathrm{X}^{2}$ & d.l. & $P$ \\
\hline Type de cI & $\begin{array}{l}\text { Violence } \\
\text { Vol }\end{array}$ & $\begin{array}{l}57,2 \\
71,4\end{array}$ & $\begin{array}{l}24,0 \\
17,1\end{array}$ & $\begin{array}{l}18,8 \\
11,4\end{array}$ & 2,63 & 2 & n.s.* \\
\hline $\begin{array}{l}\text { Sexe } \\
\text { Infracteur }\end{array}$ & $\begin{array}{l}\text { Masculin } \\
\text { Féminin } \\
\text { Mixte }\end{array}$ & $\begin{array}{l}71,7 \\
87,5 \\
76,7\end{array}$ & $\begin{array}{r}13,1 \\
0,0 \\
16,7\end{array}$ & $\begin{array}{r}15,2 \\
12,5 \\
6,7\end{array}$ & 4,44 & 4 & n.s. \\
\hline Victime & $\begin{array}{l}\text { Masculin } \\
\text { Féminin } \\
\text { Mixte }\end{array}$ & $\begin{array}{l}50,7 \\
46,6 \\
72,1\end{array}$ & $\begin{array}{l}28,4 \\
29,8 \\
23,3\end{array}$ & $\begin{array}{r}20,6 \\
23,8 \\
4,7\end{array}$ & 10,04 & 4 & .04 \\
\hline Reporter & $\begin{array}{l}\text { Masculin } \\
\text { Féminin }\end{array}$ & $\begin{array}{l}69,7 \\
70,0\end{array}$ & $\begin{array}{l}21,1 \\
20,0\end{array}$ & $\begin{array}{r}9,2 \\
10.0\end{array}$ & 0,011 & 2 & n.s. \\
\hline $\begin{array}{l}\hat{A} g e \\
\text { lnfracteur }\end{array}$ & $\begin{array}{l}-20 \\
21-30 \\
31-40 \\
41-50 \\
50 \text { et }+\end{array}$ & $\begin{array}{l}74,3 \\
72,3 \\
70,9 \\
82,5 \\
90,0\end{array}$ & $\begin{array}{r}17,1 \\
10,6 \\
9,1 \\
10,0 \\
10,0\end{array}$ & $\begin{array}{r}8.6 \\
17,0 \\
20,0 \\
7,5 \\
0,0\end{array}$ & 9,51 & 8 & n.s. \\
\hline Victime & $\begin{array}{l}-20 \\
2 l-30 \\
31-40 \\
41-50 \\
50 \mathrm{et}+\end{array}$ & $\begin{array}{l}49,2 \\
32,0 \\
36,0 \\
38,5 \\
44,1\end{array}$ & $\begin{array}{l}25,4 \\
52,0 \\
\% 6,0 \\
38,5 \\
35,3\end{array}$ & $\begin{array}{l}25,4 \\
16,0 \\
28,0 \\
23,1 \\
20,6\end{array}$ & 0,011 & 8 & n.s. \\
\hline
\end{tabular}

n.s. = non significatif, $\mathrm{P} 0,05$ 
un simple vol. Ceci correspond à ce que nous savons au sujet de la dynamique de ces types de crimes. Dans les crimes violents, la victime est plus susceptible d'avoir connu son agresseur avant le crime et d'avoir assumé un rôle plus actif dans l'événement (Wolfgang, 1958; Amir, 1971).

Les infracteurs de leur côté sont plus susceptibles de retenir davantage l'attention quand ce sont des femmes (tableau 6). En effet, il n'y avait même pas un cas où la victime a reçu plus d'attention que l'infracteur quand ce dernier était une femme. Ceci semble confirmer l'hypothèse qui veut que les événements non routiniers reçoivent plus d'attention dans les médias. En ce qui concerne le sexe du reporter nous n'avons trouvé aucune relation avec la couverture relative accordée aux victimes ou aux infracteurs.

Le tableau 6 nous révèle également une relation intéressante entre l'âge et la couverture accordée aux victimes et aux infracteurs. En effet, on assiste à une maximisation de la couverture journalistique quand l'infracteur est âgé de plus de 50 ans et que la victime est soit très jeune (moins de 20 ans), soit également âgée de plus de 50 ans. On s'intéresse également plus aux victimes quand l'infracteur $n$ 'a pas vingt ans et que la victime a entre 21 et 30 ans.

À peu près le sixième des articles faisaient référence au fait que la victime avait contribué d'une certaine façon à l'événement criminel. Comme l'indique le tableau 7 , il y a un certain nombre de relations statistiquement significatives entre nos variables indépendantes et le fait qu'on fait référence à la contribution de la victime. On retrouve de telles références beaucoup plus souvent dans les situations où l'infracteur était une femme. Cette situation est par contre inversée en ce qui concerne le sexe de la victime. Ainsi on dira beaucoup plus souvent qu'une victime mâle a contribué à sa victimisation que ce ne sera le cas pour une victime féminine. Dans les cas où il y a plusieurs victimes ou plusieurs infracteurs on fait moins référence à la contribution de la victime. Ici encore le sexe du reporter n'a pas exercé beaucoup d’influence sur la présence ou l'absence d'affirmations sur la contribution de la victime.

Dans les articles qui rapportent des crimes de violence on retrouvait beaucoup plus souvent des références à la contribution de la victime, que ce n'était le cas quand il s'agissait d'un vol simple. L'âge aussi semble avoir une certaine importance puisque les comptes rendus concernant les infracteurs et les victimes de plus de trente ans font plus souvent référence à la contribution de la victime que ce n'est le cas quand victimes ou 
TABLEAU 7

Relation entre la catégorie de crime, le sexe, Iâge et la

référence à la contribution de la victime

\begin{tabular}{|c|c|c|c|c|c|c|}
\hline & & $\begin{array}{c}\% \text { de cas avec } \\
\text { référence à la } \\
\text { contribution de la } \\
\text { victime }\end{array}$ & $\begin{array}{c}\text { \% de cas sans } \\
\text { référence à la } \\
\text { contribution de la } \\
\text { victime }\end{array}$ & $X^{2}$ & d.l. & $P$ \\
\hline Type de ci & $\begin{array}{l}\text { Violence } \\
\text { Vol }\end{array}$ & $\begin{array}{r}20,3 \\
8,6\end{array}$ & $\begin{array}{l}79,7 \\
91,4\end{array}$ & 2,77 & I & n.s.: \\
\hline Victime & $\begin{array}{l}\text { Masculin } \\
\text { Féminin } \\
\text { Mixte }\end{array}$ & $\begin{array}{r}37,6 \\
13,1 \\
9,3\end{array}$ & $\begin{array}{l}62,4 \\
86,9 \\
90,7\end{array}$ & 23,66 & 2 & 0,000 \\
\hline Reporter & $\begin{array}{l}\text { Masculin } \\
\text { Féminin }\end{array}$ & $\begin{array}{l}14,5 \\
10,0\end{array}$ & $\begin{array}{l}85,5 \\
90,0\end{array}$ & 0,15 & 1 & n.s. \\
\hline $\begin{array}{l}\text { Âge } \\
\text { Infracteur }\end{array}$ & $\begin{array}{l}-20 \\
21-30 \\
31-40 \\
41-50 \\
50 \text { et }+\end{array}$ & $\begin{array}{r}11,4 \\
8,5 \\
29,1 \\
15,0 \\
30,0\end{array}$ & $\begin{array}{l}88,6 \\
91,5 \\
70,9 \\
85,0 \\
70,0\end{array}$ & 14,12 & 4 & 0,007 \\
\hline Victime & $\begin{array}{l}-20 \\
21-30 \\
31-40 \\
41-50 \\
50+\end{array}$ & $\begin{array}{r}23,8 \\
20,0 \\
36,0 \\
7,7 \\
32,4\end{array}$ & $\begin{array}{l}76,2 \\
80,8 \\
64,0 \\
92,3 \\
67,6\end{array}$ & 4,94 & 4 & n.s. \\
\hline
\end{tabular}

${ }^{*}$ n.s. $=$ non significatif, $\mathrm{P} 0,05$ 
agresseurs sont plus jeunes. En somme le scénario où il sera fait mention de la contribution de la victime est celui où le crime est violent, où l'infracteur est une femme $d$ 'au moins trente ans et où la victime est un homme d'au moins trente ans également.

La littérature criminologique s'est longuement intéressée au fait que la participation de la victime dans les crimes de violence était plus active que dans les cas où elle n'était impliquée que dans un simple vol (Conklin, 1981). Le constat que dans les comptes rendus journalistiques, dont les femmes infracteurs sont l'objet, on ait tendance à faire allusion au rôle de la victime est en accord avec le fait que les crimes commis par les femmes ont plus tendance à être exécutés dans une situation de relation intime avec la victime, qui est généralement un homme (Conklin, 1981).

Nous avons par contre été surpris par quelques-uns de nos résultats concernant l'âge. C'est ainsi que nous avons constaté que les infracteurs plus âgés recevaient plus d'attention que les jeunes, ce qui pourrait s'expliquer par l'intérêt que portent les médias à l'inusité. Le fait que l'on s'intéresse au rôle de la victime dans un événement criminel quand l'infracteur est âgé de plus de trente ans peut également se comprendre car les crimes commis par des personnes plus âgées peuvent être plus souvent violents et incités par la victime. Cependant, on s'explique difficilement le fait que des victimes plus âgées soient considérées plus souvent comme responsables de leur victimisation que ce n'est le cas pour leurs homonymes plus jeunes. Cette perception des victimes âgées ou encore d'âge moyen ne correspond pas nécessairement à la réalité et reflète probablement la conception que se font les médias du rôle des victimes àgées comme des infracteurs.

Enfin une revue des articles classés comme analytiques aurait dû nous permettre de voir si The Citizen épousait des opinions particulières en ce qui a trait au domaine de la justice criminelle ou prônait des explications particulières dans le domaine de la criminalité. Mais les 32 articles hétérogènes retenus ne nous ont pas permis de déceler une tendance quelconque. Malgré un certain accent accordé à des problèmes qui faisaient les manchettes tels les enfants maltraités et la pornographie, la conduite en état d'ivresse et l'usage abusif de drogues, les thèmes retenus pour ces articles étaient assez hétéroclites. À peine quelques-uns faisaient allusion à la criminalité d'affaires, ou à la criminalité politique.

À peine quelques-uns des 500 articles échantillonnés étaient des éditoriaux dans lesquels on formulait une opinion. La plupart de ceux que nous avons considéré comme des articles d'ordre analytique présentaient 
soit une analyse sommaire de statistiques criminelles ou encore des entrevues avec des spécialistes ou des gens responsables dans un domaine particulier. En somme, ces dernières catégories nous mettaient en présence d'un matériel moins dogmatique et mieux équilibré, en termes d'idéologie politique.

Ce n'est que dans deux articles que les auteurs ou leurs référents posaient les problèmes des objectifs poursuivis par le système de justice criminel, c'est-à-dire ceux reliés à la dissuasion, à la réhabilitation et à la neutralisation, etc. Dans le même sens, on s'est peu intéressé aux sources de la criminalité et aux solutions qui pouvaient y être apportées. Et quand c était le cas, soit qu'on blâmait le système capitaliste et l'économie en général, soit qu'on contestait les politiques d'immigration, soit encore l'abolition de la peine capitale. En somme, on ne retrouvait pas une idéologie ou encore un point de vue dominant dans "The Citizen». Il semble, plutôt, que la tendance était de vaguer au gré de l'opinion publique en fonction des problèmes discutés et de permettre aux parties directement impliquées de se prononcer dans des entrevues.

\section{CONCLUSION}

Cinq cents articles relatifs à la criminalité, tirés d'un journal canadien à grand tirage pendant une période de deux mois, ont été retenus pour cette étude. Plus de $90 \%$ d'entre eux consistaient en rapports factuels de situations criminelles; seulement quelques-uns parmi les autres s`engageaient dans une analyse des problèmes généraux reliés à la criminalité, à ses causes et aux remèdes qu'on pourrait y apporter. Cette constatation tend à confirmer la critique que l'on adresse aux médias quand on dit qu'ils ne font au mieux qu'une analyse superficielle et que les problèmes reliés à la criminalité, bien qu'ils reçoivent une couverture considérable, ne sont pas un centre d'intérêt, mais sont plutôt utilisés comme des bouche-trous selon l'espace disponible à un moment donné (Sherizen, 1978).

Nous avons également constaté dans cette étude que l'image de la criminalité que transmet la presse écrite ne correspond pas à celle du milieu ou vivent leurs lecteurs. À preuve la forte proportion d'articles du "The Citizen" portant sur la criminalité et qui relatent des événements survenus dans d'autres villes. Il est douteux que les lecteurs distinguent constamment entre ce qui est local ou non dans l'image qu'ils se font de la criminalité. De surcroit, comme beaucoup d'autres recherches l'ont démontré, les crimes impliquant de la violence étaient surreprésentés. Ainsi, la moitié des articles traitaient de violence, ce qui appuie la thèse 
qui dit que les médias mettent l'accent sur le sensationnalisme, alors qu'en même temps les vols étaient sous-représentés, tandis que les crimes commis par l'industrie, les riches et les gens en position de pouvoir étaient largement ignorés. Donc, comme Fishman (1978) et d'autres l'ont avancé, les médias sont peut-être insidieusement cooptés par les agences officielles responsables du contrôle de la criminalité et transmettent ainsi les vues établies en ce qui concerne les paramètres de la criminalité.

Notre étude a cependant démontré, d'un autre côté, qu'on avait présenté des images assez justes des criminels et de leurs victimes. La proportion d'hommes par rapport aux femmes criminelles était élevée, ce qui correspond à ce que nous savons du sexe des infracteurs et aux découvertes de recherches déjà faites sur les médias écrits (Dussuyer, 1979). Chez les victimes cette proportion était réduite tout comme c'est le cas dans la réalité (Conklin, 1981).

En ce qui concerne les catégories d'âge, nous avons constaté que les articles présentaient une image assez exacte. Règle générale nous retrouvions de moins en moins d'articles à mesure que l'âge croissait. Il y avait cependant quelques exceptions, ainsi les adolescents délinquants étaient sous-représentés dans les articles. On peut peut-être expliquer cela tout simplement par le fait que les médias n'ont pas le droit d'identifier les jeunes délinquants. Par contre, les infracteurs et les victimes les plus âgées (plus de 50 ans) reçurent une couverture disproportionnée, ce qui nous amène à avancer que les actes criminels où ils sont impliqués sont mis en évidence à cause de l'attention qu'ils reçoivent.

Une autre indication que les médias se préoccupent beaucoup de ce qui sort de l'ordinaire et peut-être du sensationnel est le fait qu'on accorde une plus grande couverture aux infracteurs de sexe féminin. Le sexe des reporters, par contre, n'est pas apparu comme une variable importante. Comme d'autres études l'ont également démontré (Sherizen, 1978), ces derniers étaient surtout des hommes, et malgré cela il ne semble pas que cela a eu une influence sur la couverture que l'on accordait aux infracteurs ou aux victimes, pas plus que sur l'attribution des responsabilités à l'un ou à l'autre.

Le sixième des articles faisait référence au rôle de la victime dans l'événement criminel, ce qui est beaucoup moins que ce qu'ont trouvé Voumvakis et Ericson (1984) qui de leur côté évaluaient à la moitié les articles où l'essentiel du blâme était placé sur la victime. Il faut toutefois tenir compte du fait que cette étude ne portait que sur les agressions sexuelles dont étaient victimes les femmes. 
Notre étude a également constaté, qu'en ce qui concerne les crimes violents on avait beaucoup plus tendance à parler de la victime et à la blâmer, quoique nos données n'approchent pas celles de Voumakis et Ericson (1984). On ne doit pas se surprendre du fait que l'on mette davantage l'accent sur le rôle de la victime dans les crimes de violence que dans les crimes qui n'impliquent que le vol, puisque les premiers sont plus susceptibles d'impliquer une participation active de la victime (Wolgang, 1958; Amir, 1971). En ce qui concerne la possibilité que les victimes de sexe féminin soient plus souvent que les hommes blâmées pour leur rôle dans un incident criminel, notre étude a prouvé le contraire. En effet, on faisait référence beaucoup plus à la contribution de la victime dans les situations où l'infracteur était de sexe féminin et la victime masculine.

L'ambivalence des découvertes de cette recherche semble indiquer que les médias reflètent davantage la réalité dans certaines dimensions que dans d'autres. La tendance au sensationnel, au superficiel, tout comme la vision déformée de la criminalité qu'ils véhiculent indiquent que les médias sont capables d'induire en erreus le profane sur la vraie nature du problème. Par contre, on a trouvé que l'on décrivait assez fidèlement dans le détail l'identité et les rôles respectifs des infracteurs et des victimes. Il ne serait donc pas juste de rejeter catégoriquement, parce que biaisés, tous les aspects de la couverture par les médias.

\section{BIBLIOGRAPHIE}

AMIR, M. (1971), Patterns in Forcible Rape, Chicago, University of Chicago.

ANDISON, F.S. (1977), «T.V. Violence and Viewer Aggression: A Cumulation of Study Results, 1956-76», Public Opinion Quarterly 41(3): 314-331.

ANTUNES, G.E. and P.A. HURLEY (1977), "The Representation of Criminal Events in Houston's Two Daily Newspapers», Journalism Quarterly 54(4): 756-760.

CLARKE, R.V. (1983), "Situational Crime Prevention: Its Theoretical Basis and Practical Scope", in Crime and Justice: An Annual Review of Research (N. Tonry and N. Morris, édit.), Chicago, University of Chicago Press, pp. 225-256.

CONKLIN, J. (1981), Criminology, New York, MacMillan.

DAVIS, F.J. (1952), "Crime News in Colorado Newspapers", American Journal of Sociology 57 (juin), 325-330.

DOMINICK, J.R. (1978), "Crime and Law Enforcement in the Mass Media", in Deviance and Mass Media (C. Winick, édit.), Beverly Hills, Calif., Sage, pp. 105-128.

DUSSUYER, I. (1979), Crime News: A Study of 40 Ontario Newspapers, Toronto, Centre of Criminology, University of Toronto. 
EPSTEIN, E.J. (1973), News from Nowhere, New York, Random House.

EYSENCK, H.J. and D.B.K. MIAS (1978), Sex, Violence and the Media, New York, Harper and Row.

FISHMAN, M. (1978), "Crime Waves as Ideology", Social Problems 25(5), 531-543.

GABOR, T. and GOTTHEIL, E. (1984), "Offender Characteristics and Spatial Mobility: Empirical Findings and Policy Implications", Canadian Journal of Criminology $26(3), 267-281$.

GAROFALO, J. (1981), "Crime and the Mass Media: A Selective Review of Research", Journal of Research in Crime and Delinquency 18(2), 319-350.

GRABER, D.A. (1980), Crime News and the Public, New York, Praeger.

HALLORAN, J.D. (1978), "Studying Violence and the Media: A Sociological Approach», in Deviance and Mass Media (C. Winick, édit.), Beverly Hills, Calif., Sage, pp. 287-305.

HOWITT, D. and G. CUMBERBATCH (1975), Mass Media Violence and Society, London, Elek Science.

ISAACS, N.E. (1961), "The Crime of Crime Reporting", Crime and Delinquency, 7(3), 312-320.

JONES, E.T. (1976), "The Press as Metropolitan Monitor: The Case of Crimen, Public Opinion Quarterly 40(2); 239-244.

MANNHEIM, H. (1965), Comparative Criminology, Boston, Houghton Mifflin.

McLUHAN, M. (1976), "The Violence of the Media", The Canadian Forum 56(664), 9.

MOLOTCH, H. and M. LESTER (1974), "News as Purposive Behavior: The Strategic Use of Routine Events, Accidents and Scandals", American Sociological Review 39. 101-112.

PANDIANI, J.A. (1978), "Crime Time TV: If All We Knew Is What We Saw...", Contemporary Crises 2(4), 437-458.

REID, T. (1971), "Violence in Canadian TV and Newspapers», in A Media Mosaic: Canadian Communications Through a Critical Eye (W. McPayter, édit.), Toronto, Holt, Rinehart and Winston.

ROSHCO, B. (1975), Newsmaking, Chicago, University of Chicago Press.

ROYAL COMMISSION ON VIOLENCE IN THE COMMUNICATIONS INQUIRY (1976), Violence in Television, Films and News, Toronto, Thatcher.

SHERIZEN, S. (1978), "Social Creation of Crime News: All the News Fitted to Print", in Deviance and Mass Media (C. Winick, édit.), Beverly Hills, Calif., Sage, pp. 203-224.

SINGER, B.D. (1983), «Violence, Protest, and War in Television News: The United States and Canada Compared „, in Communications in Canada Society (B.d. Singer, édit.), Don Mills, Ontario, Addison-Wesley, pp. 192-196.

SOLICITOR GENERAL CANADA (1983), Canadian Urban Victimization Survey: Bulletin.

SURGEON GENERAL'S SCIENTIFIC ADVISORY COMMITTEE ON TELEVISION AND SOCIAL BEHAVIOR (1971), Television and Growing Up: The Impact of Televised Violence, Rockville, Maryland, National Institute of Mental Health. 
VOUMVAKIS, S. and R. ERICSON (1984), News Accounts of Attacks on Women: A comparison of Three Toronto Newspapers, Toronto, Centre of Criminology, University of Toronto.

WEIMANN, G. and G. FISHMAN (1984), "Attribution of Responsibility: Sex-Based Bias in Press Reports on Crime", (Unpublished Report), Haifa, Israel, Department of Sociology and Anthropology, University of Haifa.

WEIMANN, G., T. GABOR and C. BRAULT (1985), "Placing the Blame for Crime in Press Reports», Ottawa, Department of Criminology, University of Ottawa.

WOLFGANG, M. 91958, Patterns in Criminal Homicide, Philadelphia, University of Pennsylvania Press.

ZIMRING, F. (1981), «Kids, Groups and Crime: Some Implications of a Well-Known Secret", Journat of Criminal Law and Criminology, 72(3), 867-885.

\section{REMERCIEMENTS}

Les auteurs tiennent à remercier le Département de criminologie de l'Université d'Ottawa ainsi que le ministère du Solliciteur général du Canada pour leur support financier. Nous désirons également manifester notre gratitude à Tonia Barker et à Dorota Geissel, associées de recherche, pour leur précieuse aide dans la cueillette et la codification des données. 